\title{
A note on chiral fermions and heterotic strings
}

\author{
P.S. Howe \\ Department of Mathematics \\ King's College, London
}

August 6, 2018

\begin{abstract}
An off-shell, manifestly $(8,0)$ worldsheet supersymmetric, formulation of a multiplet describing physical chiral fermions is given. The multiplet can be used to complete the doubly supersymmetric (twistorlike) action for the heterotic string.
\end{abstract}


There has been a considerable amount of interest recently in reformulating the actions for supersymmetric extended objects with both world-surface and spacetime supersymmetry. This has followed on from the initial observation of Sorokin et al [1, 2] that the Brink-Schwarz [3] superparticles in three and four dimensional spacetimes admit such a reformulation with local worldline supersymmetry replacing the fermionic $\kappa$-symmmetry [1] of the BS version. A number of authors have contributed to the development of this point of view, first to superparticles in higher dimensional spacetimes, and then to heterotic strings, membranes and higher-dimensional extended objects [6, 17, 8, 9, 11, 12, 10, 13, 5, 28, 14, ?]. In addition, this new approach has much in common with various twistor-like formulations of superparticles and extended supersymmetric objects 18, 19, 22, 24, 25, 26]. One of the most interesting objects to study is undoubtedly the heterotic string in tendimensional spacetime. Up until now, a doubly supersymmetric formulation has been given for the geometrical (supergravity background) sector of the theory [16, 17] and an action proposed for the fermionic (Yang-Mills background) sector [15]. However, this latter action appears to be not completely satisfactory as the authors of 15] have themselves pointed out. It is the purpose of this letter to propose a replacement for this action which istself has some drawbacks as we shall see.

The multiplet in question is in fact known, and has appeared in the literature in different guises, for example, see [20, 23, 27]. It is an $8+8$ component multiplet which in the current context consists of 8 physical two-dimensional chiral fermions together with 8 auxiliary boson (scalar) fields. It is straightforward to write down the action in components in a flat background, and a coupling of a similar multiplet to two-dimensional supergravity (in components) has also been constructed [20]. However, this latter model has physical bosons and chiral fermions (still $8+8$ off-shell) and so cannot be taken over directly for the present purposes. In addition, the spirit of doubly supersymmetric formulations requires that the multiplet be written down in a manifestly supersymmetric fashion, i.e. in superfields. In the rest of the paper we carry this out, starting with the basic multiplet in flat superspace. We then briefly review $(8,0)$ supergeometry and adapt the multiplet to a curved background. The action we obtain is manifestly locally $(8,0)$ supersymmetric and is in addition super-Weyl invariant, although the latter property has to be established by hand. Finally we comment on the multiplet in the string context where the local worldsheet supersymmetry appears to force restric- 
tions on the Yang-Mills gauge group of the spacetime background.

Flat $(8,0)$ superspace is the $(2 \mid 8)$-dimensional (two even and eight odd) supermanifold $\mathbb{R}^{2 \mid 8}$ with standard coordinates $\left(x^{++}, x^{--}, \theta^{\alpha+}\right)$ where the signs indicate the Lorentz transformation properties of the quantities concerned. The index $\alpha$ indicates the eight-dimensional spinor representation of $S O(8)$. The supercovariant derivative $D_{\alpha+}$ satisfies

$$
\left\{D_{\alpha+}, D_{\beta+}\right\}=2 i \partial_{++}
$$

where $\partial_{++}=\frac{\partial}{\partial x^{++}}$and similarly for minuses. The multiplet of interest is a fermionic superfield $\psi_{i-}$ which satisfies the constraint

$$
D_{\alpha+} \psi_{i-}=\left(\gamma_{i}\right)_{\alpha \dot{\alpha}} P_{\dot{\alpha}}
$$

where $P_{\dot{\alpha}}$ is a bosonic superfield. The index $i(=1, \ldots 8)$ indicates the vector representation of $S O(8)$ and $\dot{\alpha}$ the dotted spinor representation (so that all three types of index, $\alpha, \dot{\alpha}, i$, run from 1 to 8). Differentiating the above constraint and using the commutation relations satisfied by the $D$ 's one finds

$$
D_{\alpha+} P_{\dot{\alpha}}=i\left(\gamma_{i}\right)_{\alpha \dot{\alpha}} \partial_{++} \psi_{i-}
$$

Further differentiation reveals that all the components of the superfield $\psi_{i-}$ are expressible in terms of the components $\psi_{i-}\left|, P_{\dot{\alpha}}\right|$ and spacetime $\left(\partial_{++}\right)$ derivatives of them, the vertical bars denoting evaluation of a superfield at $\theta=0$. Hence the above constraint defines an off-shell multiplet with $8+8$ components. The equation of motion is simply

$$
P_{\dot{\alpha}}=0
$$

which immediately implies that $\partial_{++} \psi_{i-}=0$. The component action which gives these equations is obviously the sum of the standard kinetic term for the chiral fermions together with an auxiliary term of the form $P^{2}$. However, in order to construct a superspace action it is necessary to solve the constraint (2). The solution is

$$
\psi_{i-}=\Delta_{i \dot{\alpha}(+7)} V_{\dot{\alpha}(-8)}
$$

where $V_{\dot{\alpha}(-8)}$ is the prepotential and

$$
\begin{aligned}
\Delta_{i \dot{\alpha}(+7)}= & \left(\gamma_{i}\right)_{\alpha \dot{\alpha}}\left(\tilde{D}_{\alpha(+7)}+i D_{\alpha}\left(\partial_{++}\right)^{3}\right) \\
& -\frac{i}{6}\left(\gamma_{j}\right)^{\alpha \dot{\alpha}}\left(\gamma_{i j}\right)^{\beta \gamma}\left(\tilde{D}_{\alpha \beta \gamma(+5)} \partial_{++}+i D_{\alpha \beta \gamma(+3)}\left(\partial_{++}\right)^{2}\right)
\end{aligned}
$$


The multiple derivatives are defined by

$$
\begin{aligned}
D_{\alpha_{1} \ldots \alpha_{n}(+n)} & =D_{\left[\alpha_{1}+\ldots D_{\left.\alpha_{n}\right]+}\right.} \\
\tilde{D}_{\alpha_{1} \ldots \alpha_{m}(+n)} & =\frac{1}{n !} \epsilon_{\alpha_{1} \ldots \alpha_{m} \beta_{1} \ldots \beta_{n}} D_{\beta_{1} \ldots \beta_{n}(+n)}
\end{aligned}
$$

where the square brackets denote antisymmetrisation and where, in the second equation, $m=8-n$. Since all $S O(8)$ indices are raised or lowered using the unit matrix there is no need to distinguish between upper and lower indices.

The action which gives the desired equations of motion is

$$
S=\int d^{2} x d^{8} \theta V_{\dot{\alpha}(-8)} P_{\dot{\alpha}}
$$

The Lagrangian has Lorentz weight -8 to compensate the Lorentz weight of the superspace measure. The action is invariant under the local gauge transformations

$$
\delta V_{\dot{\alpha}(-8)}=\left(\gamma_{i j k}\right)_{\dot{\alpha} \alpha} D_{\alpha+} \Lambda_{i j k(-9)}
$$

In order to couple the above system to background $(8,0)$ supergravity we briefly review the latter. We now have a general $(2 \mid 8)$ real supermanifold $M$ with local coordinates $z^{M}=\left(x^{m}, \theta^{\mu}\right)$. The structure group of $M$ is taken to be $S O(1,1) \times S O(8)$ and a set of preferred frames denoted by $E_{A}=$ $\left(E_{a}, E_{\alpha+}\right)=\left(E_{++}, E_{--}, E_{\alpha+}\right)=E_{A}^{M} \partial_{M}$. The connection form is $\Gamma_{A}^{B}$, where

$$
\begin{aligned}
& \Gamma_{\alpha+}{ }^{\beta+}=B_{\alpha}{ }^{\beta}+\delta_{\alpha}{ }^{\beta} C \\
& \Gamma_{++}{ }^{++}=2 C \\
& \Gamma_{--}{ }^{--}=-2 C
\end{aligned}
$$

are its non-vanishing components with $B$ and $C$ being the $S O(8)$ and $S O(1,1)$ parts respectively. The torsion and curvature two-forms are

$$
\begin{aligned}
& T^{A}=d E^{A}+E^{B} \Gamma_{B}{ }^{A} \\
& R_{A}^{B}=d \Gamma_{A}^{B}+\Gamma_{A}{ }^{C} \Gamma_{C}{ }^{B}
\end{aligned}
$$

where

$$
\begin{aligned}
& R_{\alpha+}{ }^{\beta+}=G_{\alpha}{ }^{\beta}+\delta_{\alpha}{ }^{\beta} H \\
& R_{++}{ }^{++}=2 H \\
& R_{--}{ }^{--}=-2 H
\end{aligned}
$$


where $G$ and $H$ are the $S O(8)$ and $S O(1,1)$ curvatures. The components of the torsion tensor are constrained to satisfy

$$
\begin{gathered}
T^{++}=i E^{\alpha+} \wedge E^{\alpha+} ; \quad T^{--}=0 \\
T^{\alpha+}=E^{--} \wedge E^{++} \Psi_{-}^{\alpha}
\end{gathered}
$$

where $E^{A}=\left(E^{++}, E^{--}, E^{\alpha+}\right)=d z^{M} E_{M}{ }^{A}$ are the basis one-forms dual to the preferred frames. The components of the curvature tensor are given by

$$
\begin{aligned}
& H_{\alpha+\beta+}=0 \\
& H_{\alpha+,++}=0 \\
& H_{\alpha+,--}=i \Psi_{\alpha-} \\
& H_{++,--}=\frac{1}{8} \nabla_{\alpha+} \Psi_{\alpha-}
\end{aligned}
$$

and

$$
\begin{aligned}
& G_{\alpha+\beta+, \gamma \delta}=0 \\
& G_{\alpha+,++, \gamma \delta}=0 \\
& G_{\alpha+,--, \gamma \delta}=-\chi_{\alpha \gamma \delta-}+2 i \delta_{\alpha[\gamma} \Psi_{\delta]-} \\
& G_{++,--, \gamma \delta}=\nabla_{[\gamma+} \Psi_{\delta-]}
\end{aligned}
$$

In the above $\nabla_{A}$ denotes the covariant derivative with respect to the $S O(8) \times$ $S O(1,1)$ structure group. The geometry is thus described by two fermionic superfields $\chi$ and $\Psi$, the former being totally antisymmetric on its $S O(8)$ indices. These superfields are themselves constrained to satisfy

$$
\nabla_{\{\alpha+} \Psi_{\beta\}-}=0
$$

and

$$
\nabla_{\alpha+} \chi_{\beta \gamma \delta}=\nabla_{[\alpha+} \chi_{\beta \gamma \delta]-}-6 i \delta_{\alpha[\beta} \nabla_{\gamma+} \Psi_{\delta]-}
$$

where the braces denote traceless symmetrisation.

The constraints are invariant under super-Weyl transformations, i.e. local rescalings of the frames; in terms of

$$
X_{A}{ }^{B}=E_{A}^{M} \delta E_{M}{ }^{A}
$$

and

$$
\Phi_{A, B}^{C}=E_{A}{ }^{M} \delta \Gamma_{M, A}^{B}
$$


the non-trivial super-Weyl transformations are given by

$$
\begin{aligned}
& X_{\alpha+}{ }^{\beta+}=\delta_{\alpha}{ }^{\beta} S \quad X_{++}{ }^{++}=X_{--}{ }^{--}=2 S \\
& X_{++}{ }^{\alpha+}=-2 i \nabla_{\alpha+} S \quad X_{--}{ }^{\alpha+}=0 \\
& \Phi_{\alpha+}=\nabla_{\alpha+} S \quad \Phi_{\alpha+, \beta \gamma}=4 \delta_{\alpha[\beta} \nabla_{\gamma]+} S+\Sigma_{\alpha \beta \gamma+} \\
& \Phi_{++}=\nabla_{++} S \quad \Phi_{--}=-\nabla_{--} S \\
& \Phi_{++, \beta \gamma}=-2 i \nabla_{\alpha \beta++} S \quad \Phi_{--, \beta \gamma}=0
\end{aligned}
$$

where $S$ is a scalar superfield parameter and $\Sigma_{\alpha \beta \gamma+}$ is totally antisymmetric. These parameters are constrained by

$$
\nabla_{\alpha+} \Sigma_{\beta \gamma \delta+}=\nabla_{[\alpha+} \Sigma_{\beta \gamma \delta]+}+6 \delta_{\alpha[\beta} \nabla_{\gamma \delta]++} S
$$

Note the similarity between this equation and equation (18) above. In fact equation (22) can be solved by

$$
\begin{aligned}
\Sigma_{\alpha \beta \gamma+} & =-2 i \nabla_{\alpha \beta \gamma(+3)} T_{--}+\sigma_{\alpha \beta \gamma+} \\
S & =\nabla_{++} T_{--}
\end{aligned}
$$

where $\sigma$ satisfies

$$
\nabla_{++} \sigma_{\alpha \beta \gamma+}=0
$$

as well as

$$
\nabla_{\alpha+} \sigma_{\beta \gamma \delta+}=\nabla_{[\alpha+} \sigma_{\beta \gamma \delta]+}
$$

One can then write $\Phi_{\alpha+, \beta \gamma}$ in the form

$$
\Phi_{\alpha+, \beta \gamma}=\nabla_{\alpha+} L_{\beta \gamma}+\sigma_{\alpha \beta \gamma+}
$$

where

$$
L_{\alpha \beta}=-2 i \nabla_{\alpha \beta++} T_{--}
$$

The component count for the parameters $S$ and $\Sigma$ therefore corresponds to an unconstrained scalar superfield together with the superfield $\sigma$, and this exactly matches the component count of the degrees of freedom present in the supergravity fields $\Psi$ and $\chi$. Thus $M$ is locally superconformally flat. In particular, the parameter $\sigma$ can be used to eliminate the $\Psi$-independent part of the solution for $\chi$ to equation (18), and we shall henceforth suppose that this has been done. 
The coupling of the chiral fermion multiplet to $(8,0)$ supergravity is almost trivial, at least as regards reparametrisations and $S O(1,1) \times S O(8)$. The constraint (2) is simply made covariant:

$$
\nabla_{\alpha+} \psi_{i-}=\left(\gamma_{i}\right)_{\alpha \dot{\alpha}} P_{\dot{\alpha}}
$$

Note that the $i$ index of $\psi$ is acted on by the local $S O(8)$ group in the vector representation. This constraint is easily verified to be consistent with the supergravity constraints; indeed, the algebra of the left covariant derivatives $\left(\nabla_{\alpha+}, \nabla_{++}\right)$is unchanged from the flat case. Since these are the only derivatives which occur in the solution, it immedately follows that flat space solution, equation 5, can be immediately generalised to the curved case provided that one replaces the ordinary derivatives in the differential operator (6) by covariant derivatives. The action is

$$
S=\int d^{2} x d^{8} \theta E V_{\dot{\alpha}(-8)} P_{\dot{\alpha}}
$$

where $E$ is the superdeterminant of the supervielbein $E_{M}{ }^{A}$. It is manifestly invariant under worldsheet superdiffeomorphisms and local $S O(1,1) \times S O(8)$ transformations. It is also invariant under chiral multiplet gauge transformations (with $\nabla$ 's) and under super-Weyl transformations which act on the chiral multiplet by

$$
\delta V_{\dot{\alpha}(-8)}=\left(-8 S+4 T_{--} \nabla_{++}+2 i \nabla_{\alpha+} T_{--} \nabla_{\alpha+}\right) V_{\dot{\alpha}(-8)}+L_{\dot{\alpha} \dot{\beta}} V_{\dot{\beta}(-8)}
$$

From this one can compute the super-Weyl variation of $\psi$ itself to be

$$
\delta \psi_{i-}=-S \psi_{i-}+L_{i j} \psi_{j-}-2 i\left(\gamma_{i}\right)_{\alpha \dot{\alpha}} \nabla_{\alpha+} T_{--} P_{\dot{\alpha}}+4 T_{--} \nabla_{++} \psi_{i-}
$$

while for $P$ one finds

$$
\delta P_{\dot{\alpha}}=L_{\dot{\alpha} \dot{\beta}} P_{\dot{\beta}}+2\left(\gamma_{i}\right)_{\dot{\alpha} \alpha} \nabla_{\alpha+} T_{--} \nabla_{++} \psi_{i-}+4 T_{--} \nabla_{++} P_{\dot{\alpha}}
$$

where we have introduced

$$
\begin{aligned}
L_{i j} & =\frac{1}{4}\left(\gamma_{i j}\right)_{\alpha \beta} L_{\alpha \beta} \\
L_{\dot{\alpha} \dot{\beta}} & =\frac{1}{4}\left(\gamma_{i j}\right)_{\dot{\alpha} \dot{\beta}} L_{i j}
\end{aligned}
$$

with $L_{\alpha \beta}$ given by (28). We note that equation (32) implies that the constraint (29) is conformally covariant, while (33) shows explicitly that the 
equation of motion $P=0$ is invariant since it implies that $\nabla_{++} \psi=0$ as well.

In the string context one requires 32 chiral fermions, and therefore four of the above multiplets. We combine them in a superfield $\psi_{i-}^{r}$ where the index $r$ runs from 1 to 4 . This index can be associated with a Yang-Mills group on the target superspace $\underline{M}$, but this group appears to be restricted to be at most $S O(4)$. The constraint now reads

$$
\hat{\nabla}_{\alpha+} \psi_{i-}^{r}=\left(\gamma_{i}\right)_{\alpha \dot{\alpha}} P_{\dot{\alpha}}^{r}
$$

where

$$
\hat{\nabla}_{A}=\nabla_{A}+A_{A}
$$

with $A$ being the pull-back onto the world-sheet of the target space YangMills field,

$$
A_{M}=\partial_{M} z^{\underline{M}} A_{\underline{M}}
$$

and where worldsheet indices are distinguished from target space indices by underlining the latter. If we apply a second covariant derivative to $\psi$ we now find a constraint on the pull-back of the Yang-Mills field strength tensor $F$; it is

$$
F_{\alpha+\beta+}=\frac{1}{8} \delta_{\alpha \beta} F_{\gamma+\gamma+}
$$

Since we wish to consider arbitrary embeddings, this implies a constraint on the target space field strength tensor which is equivalent to the equations of motion. Indeed, one can simplify matters further by using a conventional constraint on the target space so that $F_{\alpha+\beta+}=0$ on the world sheet. Given this, we find that $\left(\hat{\nabla}_{\alpha+}, \hat{\nabla}_{++}\right)$obey the same algebra as the flat derivatives and so we can simply repeat the above construction of the action in this modified case.

The above constraint on the target space gauge group is not surprising, since similar restrictions occur for $(2,0)$ and $(4,0)$ world sheet supersymmetries. It may be that a way round this problem can be found; indeed, it is not possible to couple $E_{8} \times E_{8}$ gauge fields in the $N S R$ version of the heterotic string [29] in a straightforward manner.

Acknowledgements. I thank N. Berkovits and D. Sorokin for helpful comments. 


\section{References}

[1] D.P. Sorokin, V.I. Tkach and D.V. Volkov, Mod. Phys. Lett. A4 (1989) 901.

[2] D.P. Sorokin, V.I. Tkach, D.V. Volkov and A.A. Zheltukin, Phys. Lett. 216 B (1989) 302.

[3] L. Brink and J.H. Schwarz, Phys. Lett. 100B (1981) 310.

[4] W. Siegel, Phys. Lett. 128B (1983) 397.

[5] P. Howe and P. Townsend, Phys. Lett. 259B (1991) 285.

[6] F. Delduc and E. Sokatchev, Phys. Lett. 262B (1991) 444.

[7] F. Delduc and E. Sokatchev, Class. Quant. Grav. 9 (1992) 361.

[8] F. Delduc, A. Galperin and E. Sokatchev, Nucl. Phys. B368 (1992) 143.

[9] F. Delduc, E.A. Ivanov and E. Sokatchev, Nucl. Phys. B384 (1992) 334.

[10] S. Aoyama, P. Pasti and M. Tonin, Phys. Lett. 283B (1992) 213.

[11] M. Tonin, Phys. Lett. 266B (1991) 312.

[12] M. Tonin, Int. J. Mod. Phys. 7A (1992) 6013.

[13] A. Galperin and E. Sokatchev, Phys. Rev. D46 (1992) 714.

[14] P. Pasti and M. Tonin, Padova preprint DFPD/93/TH/07 (1993).

[15] P. Pasti and M. Tonin, Padova preprint DFPD/93/TH/52 (1993).

[16] A.S. Galperin, P.S. Howe and K.S. Stelle, Nucl. Phys. B368 (1992) 248.

[17] F. Delduc, A. Galperin, P.S. Howe and E. Sokatchev, Phys. Rev. D47 (1993) 578.

[18] A. Bengtsson, I. Bengtsson, M. Cederwall and N. Linden, Phys. Rev. D36 (1987) 1766.

[19] I. Bengtsson and M. Cederwall, Nucl. Phys. B302 (1988) 81. 
[20] E. Bergshoeff, E. Sezgin and H. Nishino, Phys. Lett. 186B(1987) 167.

[21] E. Bergshoeff and E. Sezgin, Groningen-Texas AM preprint CTP TAMU-67/93, UG-5/93 (1993).

[22] N. Berkovits, Phys. Lett. 232B (1989) 184.

[23] N. Berkovits, Phys. Lett. 241B (1990) 497.

[24] N. Berkovits, Nucl. Phys. B350 (1991) 193.

[25] N. Berkovits, Nucl. Phys. B379 (1992) 96.

[26] N. Berkovits, Phys. Lett. 300B (1992) 53.

[27] A. Galperin, P.Howe and P. Townsend, Nucl. Phys. B402 (1993) 531.

[28] P.S. Howe and P.C. West, Int. J. Mod. Phys. A7 (1992) 6639.

[29] D.J. Gross, J.A. Harvey, E. Martinec and R. Rohm, Nucl. Phys. B256 (1985) 256. 